\title{
Regional models assessing region-specific determinants of low birth weight in India
}

\author{
Dharmendra Kumar Dubey ${ }^{1, *}$ and Dilip C. Nath ${ }^{2}$ \\ ${ }^{1}$ Symbiosis Institute of Health Sciences, Symbiosis International (Deemed) University, Pune 411004 , India \\ ${ }^{2}$ Assam University, Silchar 788 011, India
}

Low birth weight (LBW) is defined as birth weight less than $2.5 \mathrm{~kg}$. LBW infants remain at a relatively higher risk of mortality than those with normal birth weight. This is still a major public health problem in developing countries like India. Data from the third round of the National Family Health Survey were used for this study. The multivariable logistic regression model demonstrated a significant association between the reporting of birth weight, and education level and financial status. Furthermore, as the rate of parity increased, the odds for reporting of birth weights decreased in the northern, central and eastern regions of India.

Keywords: Heaping, low birth weight, regression models, region-specific determinants.

IN 1984, the theme for celebration of the World Health Day was 'children's health: tomorrow's wealth'. Birth weight is an important indicator of a child's vulnerability to the risk of childhood illness and chances of survival. In many developing countries, the majority of infants are not weighed at birth ${ }^{1}$. However, those who are weighed are delivered by educated mothers, who live in urban areas and have access to medical facilities, where they obtain assistance from medically trained personnel during delivery. Moreover, a study demonstrated that birth weights reported by mothers are 'heaped' in multiples of $500 \mathrm{~g}$ (ref. 2).

In the northern region of India, prevalence of low birth weight (LBW) is $40 \%$ and LBW infants are more likely to have developmental delay, poor growth and mental disabilities $^{3}$. A study conducted in a border district of Uttar Pradesh (UP) identified birth order, mode of delivery, parity and gestational period as the major risk factors for LBW, with a prevalence of 38\% (ref. 4).

In a study among the working class in Bihar, LBW prevalence of $70 \%$ was estimated, highlighting maternal age, socio-economic status, religion and occupation as associated factors 5 .

A community-based cross-sectional study in a rural block of Assam reported a prevalence of $21.8 \%$

\footnotetext{
*For correspondence. (e-mail: dubey.dharm@gmail.com)
}

highlighting the urgent need for information, education and communication (IEC) materials for identifying LBW, and to reduce its level of incidence ${ }^{6}$.

In another study conducted in Ahmedabad, it was demonstrated that reducing teenage pregnancy, improving nutrition during adolescence, increasing coverage of antenatal visits, encouraging wider birth interval and avoiding tobacco chewing, could serve as important strategies for reducing LBW in infants ${ }^{7}$. In yet another study in Ahmedabad, researchers ${ }^{7}$ found that a substantial proportion of term and preterm LBW births may be averted by improving maternal nutritional status, anaemia and antenatal care. A recent study conducted in a rural area of UP revealed that the prevalence of LBW is still high ${ }^{8}$. A hospital-based study from the central region of India (Madhya Pradesh; MP), estimated the prevalence of LBW as $36.8 \%$, and depicted gestation and parity as important maternal parameters for influencing the birth weight of an infant ${ }^{9}$.

In western India, high-risk pregnancies and mothers with $\leq 2$ years of birth spacing have been proposed as predictors of $\mathrm{LBW}^{10}$. Based on the outcome of a study conducted in Tamil Nadu, researchers emphasized the need to improve maternal health, the quality and utilization of antenatal care, weight gain during pregnancy, prevention and proper management of risk factors such as anaemia along with improving socio-economic and educational status of the mothers ${ }^{11}$.

Many hospital-based community-wide studies on LBW have been conducted in India across states; however, few region-specific studies for $\mathrm{LBW}$ have been conducted thus far. Therefore, the present study is aimed at developing regional models for assessing regional determinants of LBW, utilizing the large cross-sectional representative dataset in India.

\section{Research questions}

In view of the reviewed literature, key questions addressed in the present study are: (i) What are the region-specific patterns for reporting of infant birth weight in India? (ii) What is the prevalence of LBW at the regional level in India? (iii) What is the current status of birth weight heaping in the individual regions of India? 


\section{Objectives}

To develop regional models for assessing the regionspecific determinants of (i) birth weight reporting, and (ii) LBW in India.

\section{Data and methods}

Data from the third round of the National Family Health Survey (NFHS-3) conducted from 2005 to 2006 were utilized for this study; these are considered as secondary data. NFHS-3 was conducted to generate information on population and health indicators with special emphasis on maternal and child health outcomes, at the national and state levels. NFHS-3 is the Indian equivalent of the Demographic and Health Surveys (DHS) assessments, which used standard model questionnaires in more than 80 developing countries ${ }^{12}$. The target population for NFHS-3 in 2005-06 consisted of children aged 0-59 months ${ }^{13}$.

Birth weight was recorded in the questionnaire for births occurring during the five years preceding the survey from available reports or as reported by the mothers. NFHS-3 has been framed with robust sampling methodology. Birth weight is categorized as LBW and normal birth weight (NBW). Birth weight (LBW/NBW) is considered as outcome variable and predictor variables are described in details, which is mentioned in tables.

All 29 states were divided into six regions: northern, central, eastern, western, northeastern and southern. The states were grouped into regions as follows: northern (Jammu and Kashmir, Himachal Pradesh, Punjab, Uttarakhand, Haryana, Delhi and Rajasthan); eastern (Bihar, West Bengal, Jharkhand and Odisha); northeastern (Sikkim, Arunachal Pradesh, Nagaland, Manipur, Mizoram, Tripura, Meghalaya and Assam); western (Gujarat, Maharashtra and Goa); southern (Andhra Pradesh, Karnataka, Kerala and Tamil Nadu) and central (UP, Chhattisgarh and MP). Multivariable logistic regression model and SPSS software are used for the analysis. For the logistic regression model, birth-weight categories was considered as exposure or outcome variables and all socio-demographic and other related variables were considered as predictor variables.

\section{Results and discussion}

Here we display the datasets for the respondents' profiles, the reporting and non-reporting of birth weight, and the prevalence of LBW in the various tables listed. In the multivariable logistic regression model, outcome variables were used separately for birth-weight reporting (reported/ not-reported) and birth weight (LBW/NBW). Analysis was carried out in specific regions of the country.

Table 1 shows the region-specific profile of respondents by sub-categories of the predictor variables. Maxi- mum respondents were recorded from the central region $(16,759)$ and minimum from the northeastern region (2139). Majority of mothers residing in rural areas varied from $14.7 \%$ (northeastern region) to as large as $42.5 \%$ (western region). Moreover, the reported distribution was significantly lower in rural areas than in urban areas of all regions. Majority $(85.3 \%)$ of the respondents were residing in rural areas in the northeastern region, whereas it was only $57.5 \%$ in the western region. In Table 1 , other predictors are also elucidated in detail.

In northern region, maximum number of respondents was identified under the categories of non-working mother, which was $68.4 \%$. While the minimum number of respondents was identified under the categories of schedule tribe, which was $8.7 \%$. In the central region, it is majority $(82.8 \%)$ were Hindus and very few belonged to 'Others' category. In the eastern region, majority $(84.5 \%)$ of the respondents were residing in rural areas, and minimum (2.9\%) number of respondents belonged to 'Others' category under religion. In the north-eastern region, majority $(85.3 \%)$ of respondents were residing in rural areas, and minimum number of respondents $(5.2 \%)$ was overweight with regard to body mass index (BMI). In the western region, maximum number of respondents $(79.9 \%)$ was Hindus and number of respondents (4.3\%) were in the 35-49 years age group. In the southern region, maximum number of respondents found $(81.4 \%)$ was Hindus and the minimum number of respondents $(3.9 \%)$ with parity (5th and above).

Table 2 describes the percentage distribution of pattern for reporting birth weight, in terms of different predictors across the regions. The maximum birth weight was reported in the southern region $(72.6 \%)$ and the minimum in the central region (12.5\%). Across the various regions, the reporting of birth weight was comparatively lower in rural areas than in urban areas. Minimum reporting of birth weight was in rural areas of the central region $(8.20 \%)$, whereas maximum was recorded in rural areas of the southern region $(65.9 \%)$.

Across the northern region, maximum reporting of birth weight was found with the recall method $(91.3 \%)$ and minimum was found with the place of delivery (home; 5.5\%). Similarly, across the central, eastern and northeastern regions, maximum reporting of birth weight was found with the recall method $(90.2 \%, 89.1 \%$ and $88.0 \%$ respectively), while minimum was also found with the place of delivery (home; $3.3 \%, 5.7 \%$ and $5.1 \%$ respectively). However, in the western region, maximum reporting of birth weight was found with education (secondary and above; 95.5\%), while minimum was found with report from health card $(12.3 \%)$.

Table 3 illustrates the prevalence of LBW across the regions by predictor variables. The total maximum prevalence of LBW was found in the northern region $(27.6 \%)$ and minimum in the northeastern and southern regions ( $18.3 \%$ and $18.1 \%$ respectively). 
RESEARCH ARTICLES

Table 1. Region-specific population percentage by different background characteristics

\begin{tabular}{|c|c|c|c|c|c|c|}
\hline Variables & Northern $n(\%)$ & Central $n(\%)$ & Eastern $n(\%)$ & Northeastern $n(\%)$ & Western $n(\%)$ & Southern $n(\%)$ \\
\hline \multicolumn{7}{|l|}{ Residence } \\
\hline Urban & $2060(28.2)$ & $3436(20.5)$ & $2205(15.5)$ & $316(14.7)$ & $3000(42.5)$ & $3286(36.8)$ \\
\hline Rural & $5254(71.8)$ & $13323(79.5)$ & $12033(84.5)$ & $1824(85.3)$ & $4065(57.5)$ & $5635(63.2)$ \\
\hline \multicolumn{7}{|l|}{ Education } \\
\hline No education & $3837(53.0)$ & $10571(63.1)$ & $8332(58.5)$ & 769 (35.9) & $2135(30.2)$ & $2558(28.7)$ \\
\hline Educated but less than secondary & $2614(35.7)$ & $5065(30.2)$ & $5282(37.1)$ & $1225(57.3)$ & $4025(57.0)$ & $5084(57.0)$ \\
\hline Secondary and above & $827(11.3)$ & $1124(6.7)$ & $625(4.4)$ & $145(6.8)$ & $905(12.8)$ & $1280(14.3)$ \\
\hline \multicolumn{7}{|l|}{ Age (years) } \\
\hline $15-24$ & $2726(37.3)$ & $6462(38.6)$ & $6463(45.4)$ & $782(36.5)$ & $3180(45.0)$ & $4111(46.1)$ \\
\hline $25-34$ & $3944(53.9)$ & $8443(50.4)$ & $6528(45.8)$ & $1064(49.7)$ & $3579(50.7)$ & $4306(48.3)$ \\
\hline $35-49$ & $644(8.8)$ & $1854(11.1)$ & $1248(8.8)$ & $293(13.7)$ & $306(4.3)$ & $505(55.7)$ \\
\hline \multicolumn{7}{|l|}{ Religion } \\
\hline Hindu & $5614(76.8)$ & $13877(82.8)$ & $10674(75.0)$ & $1079(50.4)$ & 5647 (79.9) & $7261(81.4)$ \\
\hline Muslim & $1009(13.8)$ & $2774(16.6)$ & $3154(22.2)$ & $553(25.8)$ & $977(13.8)$ & $1174(13.2)$ \\
\hline Others & $691(9.8)$ & $108(0.6)$ & $411(2.9)$ & $508(23.7)$ & $440(6.2)$ & $486(5.5)$ \\
\hline \multicolumn{7}{|l|}{ Wealth index } \\
\hline Poor & $2261(30.9)$ & $9890(59.0)$ & $9245(64.9)$ & $1166(54.5)$ & $1935(27.4)$ & $2534(28.4)$ \\
\hline Middle class & $1545(21.1)$ & $3027(18.1)$ & $2307(16.2)$ & $509(23.8)$ & $1401(19.8)$ & $2392(26.8)$ \\
\hline Rich & $3508(48.0)$ & $3842(22.9)$ & $2687(18.9)$ & $465(21.7)$ & $3729(52.8)$ & $3996(44.8)$ \\
\hline \multicolumn{7}{|l|}{ Caste } \\
\hline Schedule caste & $1811(25.6)$ & $3970(23.7)$ & $2837(20.9)$ & $243(14.3)$ & $1150(16.5)$ & $1728(20.1)$ \\
\hline Schedule tribe & $618(8.7)$ & $1451(8.7)$ & $1406(10.4)$ & $565(33.4)$ & $838(12.0)$ & $512(5.9)$ \\
\hline Other backward class & $2198(31.1)$ & $8138(48.6)$ & $5187(38.3)$ & $319(18.8)$ & $2219(31.8)$ & $4901(56.9)$ \\
\hline General & $2444(34.6)$ & $3175(19.0)$ & $4120(30.4)$ & $566(33.4)$ & $2775(39.7)$ & $1479(17.2)$ \\
\hline \multicolumn{7}{|l|}{ Place of delivery } \\
\hline Home & $4541(62.1)$ & $13139(78.4)$ & $10148(71.3)$ & $1559(72.9)$ & $2776(39.3)$ & $2298(25.8)$ \\
\hline Institutional & $2773(37.9)$ & $3620(21.6)$ & $4091(28.7)$ & $581(27.1)$ & $4288(60.7)$ & $6624(74.2)$ \\
\hline \multicolumn{7}{|l|}{ Birth order } \\
\hline First & $2247(30.7)$ & $3880(23.2)$ & $4247(29.8)$ & $682(31.9)$ & $2569(36.4)$ & $3481(39.0)$ \\
\hline Second & $1976(27.0)$ & $3713(22.2)$ & $3524(24.8)$ & $542(25.3)$ & 2253 (31.9) & $3281(36.8)$ \\
\hline Third & $1246(17.0)$ & $2864(17.1)$ & $2273(16.0)$ & $336(15.7)$ & $1117(15.8)$ & $1304(14.6)$ \\
\hline Fourth & $733(10.0)$ & $2127(12.7)$ & $1596(11.2)$ & $238(11.1)$ & $548(7.8)$ & $511(5.7)$ \\
\hline Fifth and above & $1112(15.2)$ & $4174(24.9)$ & $2600(18.3)$ & $341(16.0)$ & $578(8.2)$ & $344(3.9)$ \\
\hline \multicolumn{7}{|l|}{ Anaemia } \\
\hline Anaemic mothers & $3980(56.5)$ & 8933 (57.9) & $9612(69.8)$ & $1344(68.8)$ & $3644(56.3)$ & $4827(57.7)$ \\
\hline Mothers not anaemic & $3061(43.5)$ & $6508(42.1)$ & $4149(30.2)$ & $609(31.2)$ & $2833(43.7)$ & $3546(42.3)$ \\
\hline \multicolumn{7}{|l|}{ Body mass index } \\
\hline Normal & $4058(57.1)$ & $8941(56.7)$ & $7156(51.2)$ & $1257(60.8)$ & $3369(50.4)$ & $4559(53.2)$ \\
\hline Underweight & $2396(33.7)$ & $6096(38.7)$ & $6338(45.4)$ & $702(34.0)$ & $2694(40.3)$ & $2805(32.7)$ \\
\hline Overweight & $649(9.1)$ & $721(4.6)$ & $476(3.4)$ & $107(5.2)$ & $615(9.2)$ & $1211(14.1)$ \\
\hline \multicolumn{7}{|l|}{ Working status } \\
\hline Non-working & $4996(68.4)$ & $11982(71.6)$ & $10704(75.2)$ & $1615(75.7)$ & $4479(63.6)$ & $5980(67.2)$ \\
\hline Working & $2308(31.6)$ & $4747(28.4)$ & $3522(24.8)$ & $518(24.3)$ & $2565(36.4)$ & $2923(32.8)$ \\
\hline Total & $7314(13.0)$ & $16759(29.7)$ & $14239(25.2)$ & $2139(3.8)$ & $7065(12.5)$ & 8922 (15.8) \\
\hline
\end{tabular}

Across the northern region, maximum prevalence of LBW was found with schedule caste $(36.2 \%)$ and minimum was found with the level of education of the mothers (secondary and above; 21.8\%). In the central region, maximum and minimum prevalence of LBW were both found with age (15-24 years; $28.2 \%$ and $35-49$ years; $10.1 \%)$. In the eastern region, maximum prevalence of LBW was found with parity (fifth and above; $31.4 \%$ ), whereas the minimum was found with the level of education (secondary and above; $13.3 \%$ ). In the northeastern region, the maximum and minimum prevalence of LBW were both found with education (uneducated; $27.4 \%$, and secondary and above; $9.7 \%$ ). In the western region, maximum prevalence of $\mathrm{LBW}$ was found with parity (fourth order; 27.9\%), whereas minimum was found with education (secondary and above; 16.2\%). In the southern region, maximum prevalence of LBW was found with parity (fifth and above; 24.1\%), whereas 
RESEARCH ARTICLES

Table 2. Birth weight reported by region-specific population in India according to different background characteristics

\begin{tabular}{|c|c|c|c|c|c|c|}
\hline Predictors & Northern $n(\%)$ & Central $n(\%)$ & Eastern $n(\%)$ & Northeast $n(\%)$ & Western $n(\%)$ & Southern $n(\%)$ \\
\hline \multicolumn{7}{|l|}{ Residence } \\
\hline Urban & $2060(50.4)$ & $3436(29.2)$ & $2205(55.8)$ & $315(60.3)$ & $3000(79.7)$ & $3287(84.2)$ \\
\hline Rural & $5254(19.0)$ & $13323(8.2)$ & $12033(19.2)$ & $1824(20.1)$ & $4065(52.6)$ & $5635(65.9)$ \\
\hline \multicolumn{7}{|l|}{ Education } \\
\hline No education & $3873(12.1)$ & $10571(5.3)$ & $8332(10.8)$ & $768(9.5)$ & $2135(38.4)$ & $2559(44.1)$ \\
\hline Educated but less than secondary & $2614(35.2)$ & $5064(18.4)$ & $5282(40.3)$ & $1225(30.3)$ & $4025(70.0)$ & $5084(80.8)$ \\
\hline Secondary and above & $828(78.3)$ & $1123(54.3)$ & $625(82.7)$ & $145(77.9)$ & $904(95.5)$ & $1280(97.3)$ \\
\hline \multicolumn{7}{|l|}{ Age (years) } \\
\hline $15-24$ & $2727(27.8)$ & $6462(12.7)$ & $6463(27.3)$ & $782(24.4)$ & $3180(64.2)$ & $4110(69.3)$ \\
\hline $25-34$ & $3944(30.0)$ & 8443 (13.4) & $6528(25.2)$ & $1064(27.9)$ & $3579(64.4)$ & $4306(76.2)$ \\
\hline $35-49$ & $644(14.8)$ & $1853(7.9)$ & $1248(10.9)$ & $293(23.5)$ & $306(59.5)$ & $505(69.9)$ \\
\hline \multicolumn{7}{|l|}{ Religion } \\
\hline Hindu & $5615(27.6)$ & $13877(12.6)$ & $10674(27.9)$ & $1079(35.1)$ & $5647(62.8)$ & $7261(70.9)$ \\
\hline Muslim & $1008(16.1)$ & $2774(10.9)$ & $3154(15.9)$ & $553(7.2)$ & $977(70.0)$ & $1174(79.4)$ \\
\hline Others & $691(47.6)$ & $108(47.2)$ & $411(16.5)$ & $508(27.4)$ & $440(66.8)$ & $487(82.8)$ \\
\hline \multicolumn{7}{|l|}{ Wealth index } \\
\hline Poor & $2261(8.4)$ & $9890(5.7)$ & 9245 (14.2) & $1166(8.3)$ & $1935(38.2)$ & $2534(49.4)$ \\
\hline Middle class & $1545(15.4)$ & $3026(9.9)$ & 2307 (29.9) & $509(31.6)$ & $1400(52.4)$ & $2392(68.2)$ \\
\hline Rich & $3508(45.9)$ & $3843(32.2)$ & $2686(57.4)$ & $464(64.4)$ & 3729 (81.9) & $3996(90.0)$ \\
\hline \multicolumn{7}{|l|}{ Caste } \\
\hline Schedule caste & $1811(20.2)$ & $3970(8.2)$ & $2836(24.8)$ & $242(29.3)$ & $1151(59.3)$ & $1729(68.5)$ \\
\hline Schedule tribe & $618(12.9)$ & $1451(9.2)$ & $1406(15.1)$ & $565(31.7)$ & $838(39.0)$ & $511(40.9)$ \\
\hline Other backward class & $2198(23.9)$ & $8138(11.1)$ & $5187(16.6)$ & $319(31.7)$ & $2219(63.7)$ & $4901(73.8)$ \\
\hline General & $2444(42.6)$ & $3176(23.1)$ & $4120(39.2)$ & $566(26.9)$ & $2775(74.5)$ & $1480(82.3)$ \\
\hline \multicolumn{7}{|l|}{ Place of delivery } \\
\hline Home & $4541(5.5)$ & $13139(3.3)$ & $10148(5.7)$ & $1559(5.1)$ & $2776(23.0)$ & $2298(23.0)$ \\
\hline Institutional & $2773(64.5)$ & $3620(45.9)$ & $4091(72.5)$ & $581(82.3)$ & $4288(90.7)$ & $6624(89.8)$ \\
\hline \multicolumn{7}{|l|}{ Birth order } \\
\hline First & $2246(41.9)$ & $3881(22.6)$ & $4247(40.5)$ & $682(40.9)$ & $2569(74.7)$ & $3481(81.0)$ \\
\hline Second & $1976(33.0)$ & $3713(17.0)$ & $3524(29.2)$ & $542(28.8)$ & $2253(66.4)$ & $3280(73.7)$ \\
\hline Third & $1246(19.7)$ & $2864(8.6)$ & $2273(17.8)$ & $336(19.0)$ & $1117(56.1)$ & $1304(62.7)$ \\
\hline Fourth & $733(13.5)$ & $2127(7.3)$ & $1595(12.2)$ & $238(13.4)$ & $547(49.0)$ & $511(52.6)$ \\
\hline Fifth and above & $1112(8.9)$ & $4174(4.6)$ & $2599(7.5)$ & $341(7.6)$ & $578(37.5)$ & $344(45.9)$ \\
\hline \multicolumn{7}{|l|}{ Anaemia } \\
\hline Anaemic mothers & $3980(23.2)$ & 8933 (11.3) & $9612(22.8)$ & $1344(24.8)$ & $3644(60.5)$ & $4827(70.3)$ \\
\hline Mother not anaemic & $3060(33.0)$ & $6508(14.7)$ & $4149(29.3)$ & $609(31.2)$ & $2834(67.9)$ & $3546(77.8)$ \\
\hline \multicolumn{7}{|l|}{ Body mass index } \\
\hline Normal & $4058(27.1)$ & $8942(13.1)$ & $7156(27.6)$ & $1257(27.0)$ & $3369(66.0)$ & $4559(75.0)$ \\
\hline Underweight & $2397(21.0)$ & $6096(9.5)$ & $6338(19.1)$ & 703 (19.5) & $2694(55.7)$ & $2805(62.2)$ \\
\hline Overweight & $649(55.3)$ & $721(35.1)$ & $476(61.3)$ & $107(59.8)$ & $615(86.7)$ & $1211(91.4)$ \\
\hline \multicolumn{7}{|l|}{ Working status } \\
\hline Non-working & $4996(31.9)$ & $11982(13.3)$ & $10704(27.5)$ & $1615(26.3)$ & $4479(71.0)$ & $5981(78.4)$ \\
\hline Working & $2308(19.0)$ & $4748(10.6)$ & $3522(16.9)$ & $518(25.5)$ & $2565(52.2)$ & $2923(61.0)$ \\
\hline \multicolumn{7}{|l|}{ Methods of reporting } \\
\hline From card & $177(8.7)$ & $205(9.8)$ & $386(10.9)$ & $67(12.0)$ & $555(12.3)$ & $1365(21.1)$ \\
\hline From recall & $1861(91.3)$ & $1895(90.2)$ & $3159(89.1)$ & $490(88.0)$ & $3973(87.7)$ & $5115(78.9)$ \\
\hline Total & $7314(27.9)$ & $16759(12.5)$ & $14239(24.9)$ & $2140(26.0)$ & $7064(64.1)$ & $8922(72.6)$ \\
\hline
\end{tabular}

minimum was found with the level of education (secondary and above; $10.9 \%$ ).

Table 4 shows the results of different birth weight reporting values across the regions and adjusted odds ratio $(P<0.05)$. In the northern region, the rural respondents were $35 \%$ less likely to report birth weight than their urban counterparts. Respondents who were educated (44\%) were four times more likely to report birth weight than the uneducated group. Respondents in the age group of 25-34 years $(23 \%)$ were more likely to report birth weight than those in the age group of 15-24 years. Respondents belonging to other religions were $41 \%$ more 
Table 3. Region-specific population percentage of low birth weight according to different background characteristics in India

\begin{tabular}{|c|c|c|c|c|c|c|c|}
\hline Predictors & $\begin{array}{c}\text { Northern } \\
n(\%)\end{array}$ & $\begin{array}{c}\text { Central } \\
n(\%)\end{array}$ & $\begin{array}{c}\text { Eastern } \\
n(\%)\end{array}$ & $\begin{array}{c}\text { Northeastern } \\
n(\%)\end{array}$ & $\begin{array}{l}\text { Western } \\
n(\%)\end{array}$ & $\begin{array}{c}\text { Southern } \\
n(\%)\end{array}$ & $\begin{array}{l}\text { India } \\
n(\%)\end{array}$ \\
\hline \multicolumn{8}{|l|}{ Residence } \\
\hline Urban & $1038(25.8)$ & $1004(21.5)$ & $1231(21.1)$ & $190(16.3)$ & $2392(19.8)$ & $2768(15.1)$ & $1668(19.3)$ \\
\hline Rural & $999(29.5)$ & $1096(25.2)$ & $2314(24.1)$ & $367(19.1)$ & $2137(24.7)$ & $3711(20.3)$ & $2478(23.3)$ \\
\hline \multicolumn{8}{|l|}{ Education } \\
\hline No education & $470(34.0)$ & $556(23.6)$ & $897(28.9)$ & $73(27.4)$ & $819(27.2)$ & $1229(21.3)$ & $1034(26.2)$ \\
\hline Educated but less than secondary & $1920(28.50)$ & $935(24.5)$ & $2131(22.9)$ & $371(18.9)$ & $2846(22.4)$ & $4107(19.4)$ & $2483(22.0)$ \\
\hline Secondary and above & $648(21.8)$ & $611(21.8)$ & $517(13.3)$ & $113(9.7)$ & $863(16.2)$ & $1245(10.9)$ & $629(15.7)$ \\
\hline \multicolumn{8}{|l|}{ Age (years) } \\
\hline $15-24$ & $758(30.9)$ & $820(28.2)$ & $1766(26.0)$ & $191(21.5)$ & $2041(24.8)$ & $2847(19.3)$ & $2021(24.0)$ \\
\hline $25-34$ & $1185(25.7)$ & $1133(21.8)$ & $1643(20.0)$ & $297(16.2)$ & $2305(19.5)$ & $3280(17.7)$ & $1960(19.9)$ \\
\hline $35-49$ & $95(25.3)$ & $148(10.1)$ & $136(21.3)$ & $70(18.6)$ & $182(24.2)$ & $354(11.6)$ & $165(16.8)$ \\
\hline \multicolumn{8}{|l|}{ Religion } \\
\hline Muslim & $162(26.5)$ & $302(21.5)$ & $501(27.5)$ & $40(25.0)$ & $685(19.4)$ & $932(15.0)$ & $529(20.2)$ \\
\hline Others & $329(25.8)$ & $51(17.6)$ & $68(16.2)$ & $138(13.8)$ & $294(25.5)$ & $403(16.6)$ & $267(20.8)$ \\
\hline \multicolumn{8}{|l|}{ Wealth index } \\
\hline Poor & $189(30.2)$ & $565(25.1)$ & $1314(27.3)$ & 97 (24.7) & $740(27.3)$ & $1252(21.7)$ & $1055(25.4)$ \\
\hline Middle class & $238(33.2)$ & $301(25.2)$ & $690(25.4)$ & $161(22.4)$ & $733(25.6)$ & $1632(20.6)$ & $889(23.7)$ \\
\hline Rich & $1611(26.5)$ & $1236(22.2)$ & $1543(18.4)$ & $300(13.7)$ & $3055(20.0)$ & $3597(15.7)$ & $2202(19.4)$ \\
\hline \multicolumn{8}{|l|}{ Caste } \\
\hline Schedule caste & $366(28.7)$ & $325(21.2)$ & $705(25.0)$ & $71(23.9)$ & $682(24.6)$ & $1185(21.1)$ & $784(23.5)$ \\
\hline Schedule tribe & $80(36.2)$ & $133(24.1)$ & $212(22.6)$ & $178(14.6)$ & $327(26.0)$ & $209(22.0)$ & $266(23.3)$ \\
\hline Other backward class & $525(31.0)$ & $907(24.7)$ & $858(22.7)$ & $101(22.8)$ & $1413(23.1)$ & 3617 (17.9) & $1578(21.3)$ \\
\hline General & $1040(24.6)$ & $733(22.8)$ & $1615(22.1)$ & $151(15.2)$ & $2065(20.0)$ & $1218(15.3)$ & $1402(20.6)$ \\
\hline \multicolumn{8}{|l|}{ Place of delivery } \\
\hline Home & $250(32.4)$ & 439 (19.8) & $578(22.0)$ & $79(20.3)$ & $639(25.8)$ & $529(24.0)$ & $604(24.0)$ \\
\hline Institutional & $1788(27.0)$ & $1661(24.4)$ & $2967(23.3)$ & $478(17.8)$ & $3889(21.5)$ & $5951(17.6)$ & $3537(21.2)$ \\
\hline First & $942(26.0)$ & $876(27.1)$ & $1722(24.2)$ & $279(19.7)$ & $1919(24.4)$ & $2819(17.4)$ & $1912(22.3)$ \\
\hline Second & $653(26.8)$ & $631(21.4)$ & $1029(20.7)$ & $156(17.3)$ & $1497(19.1)$ & $2416(17.6)$ & $1261(19.8)$ \\
\hline Third & $245(31.4)$ & $245(21.6)$ & $404(22.0)$ & $64(14.1)$ & $627(18.7)$ & $818(20.5)$ & $513(21.3)$ \\
\hline Fourth & $99(34.3)$ & $155(16.1)$ & $195(19.0)$ & $32(15.6)$ & $269(27.9)$ & $269(18.6)$ & $226(22.2)$ \\
\hline Fifth and above & $99(32.3)$ & $195(22.1)$ & $194(31.4)$ & $26(19.2)$ & $217(25.3)$ & $158(24.1)$ & $234(26.4)$ \\
\hline \multicolumn{8}{|l|}{ Anaemia } \\
\hline Anaemic mothers & $923(30.7)$ & $1010(25.0)$ & $2190(24.5)$ & $333(20.4)$ & $2204(23.0)$ & $3394(18.6)$ & $2277(22.6)$ \\
\hline Mothers not anaemic & $1010(25.4)$ & $959(22.2)$ & $1215(21.4)$ & $190(15.8)$ & $1923(22.4)$ & $2758(17.4)$ & $1670(20.7)$ \\
\hline \multicolumn{8}{|l|}{ Body mass index } \\
\hline Normal & $1099(26.9)$ & $11168(24.1)$ & $1976(21.3)$ & $339(15.3)$ & $2225(21.5)$ & $3421(17.3)$ & $2119(20.7)$ \\
\hline Underweight & $502(32.9)$ & $579(25.6)$ & $1211(28.5)$ & $137(28.5)$ & $1500(25.5)$ & $1743(21.5)$ & $1454(25.6)$ \\
\hline Overweight & $358(23.7)$ & $252(15.9)$ & $292(15.1)$ & $63(14.3)$ & $533(18.4)$ & $1107(15.4)$ & $448(17.2)$ \\
\hline \multicolumn{8}{|l|}{ Working status } \\
\hline Non-working & $1595(27.5)$ & $1598(24.3)$ & $2947(23.2)$ & $423(18.20)$ & $3179(22.1)$ & $4688(17.4)$ & $3108(21.5)$ \\
\hline Working & $439(28.0)$ & $501(20.4)$ & $595(22.4)$ & $133(18.0)$ & $1339(22.2)$ & $1783(19.8)$ & $1032(21.5)$ \\
\hline \multicolumn{8}{|l|}{ Reporting method } \\
\hline From card & 177 (31.60) & $206(23.3)$ & $386(20.5)$ & $68(20.6)$ & $555(23.8)$ & $1365(14.8)$ & $979(35.5)$ \\
\hline From recall & $1861(27.2)$ & $1895(23.5)$ & $3160(23.4)$ & $490(18.0)$ & $3973(21.8)$ & $5116(19.0)$ & $6756(41.0)$ \\
\hline Total & 2038 (27.6) & $2101(23.4)$ & $3546(23.0)$ & $558(18.3)$ & $4528(22.1)$ & $6481(18.1)$ & $4146(21.5)$ \\
\hline
\end{tabular}

likely to report birth weight than Hindus. Rich respondents were $57 \%$ more likely to report birth weight than their poor counterparts. Respondents who had delivered in institutions were 20 times more likely to report birth weight than those who delivered at home. Mothers experiencing third birth, fourth birth, and fifth birth and above were
$28 \%, 34 \%$ and $32 \%$ less likely to report birth weight respectively, than those experiencing first birth. In the table, details are given according to the various regions.

Table 5 shows the results of multivariable logistic regression of outcome variable birth weight (LBW and NBW) across the regions, and the adjusted odds ratio 
RESEARCH ARTICLES

Table 4. Region-wise multivariable logistic regression results between non-reporting and reporting of birth weight by different background characteristics

\begin{tabular}{|c|c|c|c|c|c|c|}
\hline Predictors & Northern & Central & Eastern & Northeastern & Western & Southern \\
\hline Residence & $\operatorname{Odds}(\mathrm{p})$ & $\operatorname{Odds}(\mathrm{p})$ & $\operatorname{Odds}(\mathrm{p})$ & $\operatorname{Odds}(\mathrm{p})$ & $\operatorname{Odds}(\mathrm{p})$ & $\operatorname{Odds}(\mathrm{p})$ \\
\hline Urban & Ref. & Ref. & Ref. & Ref. & Ref. & Ref. \\
\hline \multicolumn{7}{|l|}{ Education } \\
\hline No education & Ref. & Ref. & Ref. & Ref. & Ref. & Ref. \\
\hline Educated but less than secondary & $1.44(0.0)$ & $2.18(0.0)$ & $2.52(0.0)$ & $2.17(0.0)$ & $1.73(0.0)$ & $2(0.0)$ \\
\hline Secondary and above & $4.29(0.0)$ & $3.14(0.0)$ & $5.72(0.0)$ & $3.36(0.01)$ & $6.78(0.0)$ & $6.06(0.0)$ \\
\hline \multicolumn{7}{|l|}{ Age (years) } \\
\hline $15-24$ & Ref. & Ref. & Ref. & Ref. & Ref. & Ref. \\
\hline $25-34$ & $1.23(0.03)$ & $1.20(0.02)$ & $1(0.97)$ & $1.27(0.30)$ & $1.05(0.57)$ & $1.17(0.05)$ \\
\hline $35-49$ & $0.95(0.80)$ & $1.38(0.03)$ & $0.83(0.26)$ & $1.19(0.65)$ & $1.27(0.26)$ & $1.04(0.82)$ \\
\hline \multicolumn{7}{|l|}{ Religion } \\
\hline Hindu & Ref. & Ref. & Ref. & Ref. & Ref. & Ref. \\
\hline Others & $1.41(0.01)$ & $1.66(0.07)$ & $0.80(0.28)$ & $0.86(0.61)$ & $1.31(0.11)$ & $0.65(0.01)$ \\
\hline \multicolumn{7}{|l|}{ Wealth index } \\
\hline Poor & Ref. & Ref. & Ref. & Ref. & Ref. & Ref. \\
\hline Middle class & $1.10(0.45)$ & $1.03(0.75)$ & $0.95(0.55)$ & $1.95(0.0)$ & $0.93(0.49)$ & $1.56(0.0)$ \\
\hline Rich & $1.57(0.0)$ & $1.53(0.0)$ & $1.09(0.32)$ & $2.70(0.0)$ & $1.61(0.0)$ & $2.41(0.0)$ \\
\hline \multicolumn{7}{|l|}{ Caste } \\
\hline Schedule caste & Ref. & Ref. & Ref. & Ref. & Ref. & Ref. \\
\hline Schedule tribe & $0.82(0.27)$ & $2.74(0.0)$ & $1.21(0.12)$ & $1.99(0.06)$ & $1.69(0.0)$ & $0.73(0.03)$ \\
\hline Other backward class & $1.17(0.14)$ & $1.20(0.04)$ & $0.51(0.0)$ & $0.91(0.78)$ & $1.57(0.0)$ & $0.83(0.03)$ \\
\hline General & $1.07(0.54)$ & $1.19(0.09)$ & $1.15(0.12)$ & $0.54(0.05)$ & $1.59(0.0)$ & $0.82(0.12)$ \\
\hline \multicolumn{7}{|l|}{ Place of delivery } \\
\hline Home & Ref. & Ref. & Ref. & Ref. & Ref. & Ref. \\
\hline Institutional & $19.76(0.0)$ & $14.60(0.0)$ & $24.61(0.0)$ & $53.61(0.0)$ & $20.44(0.0)$ & $17.89(0.0)$ \\
\hline Second & $0.96(0.68)$ & $0.87(0.08)$ & $0.81(0.01)$ & $0.88(0.61)$ & $0.97(0.75)$ & $0.84(0.04)$ \\
\hline Third & $0.72(0.01)$ & $0.51(0.0)$ & $0.7(0.01)$ & $0.77(0.41)$ & $0.86(0.20)$ & $0.80(0.04)$ \\
\hline Fourth & $0.66(0.01)$ & $0.54(0.0)$ & $0.67(0.0)$ & $0.63(0.26)$ & $0.89(0.46)$ & $0.70(0.02)$ \\
\hline Fifth and above & $0.68(0.03)$ & $0.42(0.0)$ & $0.53(0.0)$ & $0.48(0.09)$ & $0.62(0.0)$ & $0.64(0.02)$ \\
\hline \multicolumn{7}{|l|}{ Anaemia } \\
\hline Anaemic mothers & Ref. & Ref. & Ref. & Ref. & Ref. & Ref. \\
\hline Mother not anaemic & $1.24(0.01)$ & $1.04(0.43)$ & $1.21(0.0)$ & $0.95(0.78)$ & $1.13(0.11)$ & $0.97(0.67)$ \\
\hline \multicolumn{7}{|l|}{ Body mass index } \\
\hline Normal & Ref. & Ref. & Ref. & Ref. & Ref. & Ref. \\
\hline Underweight & $1.08(0.35)$ & $0.88(0.05)$ & $0.93(0.25)$ & $0.88(0.56)$ & $1.05(0.56)$ & $0.81(0.01)$ \\
\hline Overweight & $1.14(0.30)$ & $1.14(0.26)$ & $0.97(0.81)$ & $1.25(0.60)$ & $1.26(0.15)$ & $1.06(0.69)$ \\
\hline \multicolumn{7}{|l|}{ Working status } \\
\hline Non-working & Ref. & Ref. & Ref. & Ref. & Ref. & Ref. \\
\hline Working & $0.93(0.43)$ & $1.66(0.0)$ & $1.28(0.0)$ & $1.21(0.41)$ & $0.86(0.06)$ & $1.28(0.0)$ \\
\hline
\end{tabular}

$(P<0.05)$. In the Northern region, respondents with secondary education and above were $34 \%$ less likely to deliver LBW infants than those uneducated.

In the central region, respondents of the age groups of 35-49 years $(69 \%)$ were less likely to deliver LBW infants than those of the age group of 15-24 years.

Respondents who delivered in institutions were $52 \%$ more likely to give birth to LBW infants than those who delivered at home. Overweight mothers were 38\% less likely to deliver infants with LBW than normal and underweight mothers.

In the eastern region, $24 \%$ of the respondents were educated up to the secondary level, whereas $48 \%$ were educated above secondary level. The educated were more likely to deliver LBW infants than the uneducated. Respondents of the age group of 25-34 years were $20 \%$ less likely to give birth to infants with LBW than those in the age group of 15-24 years. Respondents who delivered 
RESEARCH ARTICLES

Table 5. Region-specific multivariable logistic regression results between normal birth weight and low birth weight by different predictors

\begin{tabular}{|c|c|c|c|c|c|c|}
\hline Predictors & Northern & Central & Eastern & Northeastern & Western & Southern \\
\hline Residence & $\operatorname{Odds}(\mathrm{p})$ & $\operatorname{Odds}(\mathrm{p})$ & $\operatorname{Odds}(\mathrm{p})$ & $\operatorname{Odds}(\mathrm{p})$ & $\operatorname{Odds}(\mathrm{p})$ & $\operatorname{Odds}(\mathrm{p})$ \\
\hline Urban & Ref. & Ref. & Ref. & Ref. & Ref. & Ref. \\
\hline Rural & $0.99(0.95)$ & $1.19(0.20)$ & $0.94(0.59)$ & $0.74(0.31)$ & $1.05(0.61)$ & $1.25(0.0)$ \\
\hline \multicolumn{7}{|l|}{ Education } \\
\hline No education & Ref. & Ref. & Ref. & Ref. & Ref. & Ref. \\
\hline Educated but less than secondary & $0.78(0.11)$ & $1.03(0.85)$ & $0.76(0.01)$ & $0.65(0.23)$ & $0.87(0.21)$ & $1.03(0.76)$ \\
\hline Secondary and above & $0.66(0.03)$ & $1.12(0.56)$ & $0.52(0.0)$ & $0.36(0.06)$ & $0.65(0.0)$ & $0.59(0.0)$ \\
\hline \multicolumn{7}{|l|}{ Age (years) } \\
\hline $15-24$ & Ref. & Ref. & Ref. & Ref. & Ref. & Ref. \\
\hline $25-34$ & $0.79(0.06)$ & $0.81(0.10)$ & $0.80(0.02)$ & $0.91(0.73)$ & $0.85(0.07)$ & $0.95(0.49)$ \\
\hline $35-49$ & $0.77(0.35)$ & $0.31(0.0)$ & $0.74(0.24)$ & $1.17(0.75)$ & $1.26(0.26)$ & $0.55(0.0)$ \\
\hline \multicolumn{7}{|l|}{ Religion } \\
\hline Hindu & Ref. & Ref. & Ref. & Ref. & Ref. & Ref. \\
\hline Others & $0.99(0.93)$ & $0.93(0.85)$ & $0.86(0.66)$ & $0.96(0.93)$ & $1.26(0.26)$ & $1.16(0.35)$ \\
\hline \multicolumn{7}{|l|}{ Wealth index } \\
\hline Poor & Ref. & Ref. & Ref. & Ref. & Ref. & Ref. \\
\hline Middle class & $1.35(0.18)$ & $0.92(0.64)$ & $0.96(0.75)$ & $0.98(0.96)$ & $1.04(0.79)$ & $1.03(0.74)$ \\
\hline Rich & $1.32(0.21)$ & $0.83(1.19)$ & $0.75(0.03)$ & $0.66(0.29)$ & $0.88(0.32)$ & $0.97(0.76)$ \\
\hline \multicolumn{7}{|l|}{ Caste } \\
\hline Schedule caste & Ref. & Ref. & Ref. & Ref. & Ref. & Ref. \\
\hline Schedule tribe & $1.58(0.11)$ & $1.27(0.37)$ & $1(1)$ & $0.66(0.37)$ & $1.20(0.30)$ & $1.01(0.97)$ \\
\hline Other backward class & $1.27(0.13)$ & $1.35(0.08)$ & $1.03(0.80)$ & $0.99(0.98)$ & $1.10(0.47)$ & $0.91(0.27)$ \\
\hline General & $1.08(0.61)$ & $1.19(0.34)$ & $1.04(0.73)$ & $0.66(0.28)$ & $0.98(0.88)$ & $0.86(0.21)$ \\
\hline \multicolumn{7}{|l|}{ Place of delivery } \\
\hline Home & Ref. & Ref. & Ref. & Ref. & Ref. & Ref. \\
\hline Institutional & $0.81(0.19)$ & $1.52(0.01)$ & $1.46(0.0)$ & $1.05(0.90)$ & $0.92(0.47)$ & $0.83(0.10)$ \\
\hline Second & $1.08(0.56)$ & $0.90(0.45)$ & $0.88(0.21)$ & $0.95(0.86)$ & $0.72(0.0)$ & $0.99(0.89)$ \\
\hline Third & $1.32(0.12)$ & $0.98(0.89)$ & $0.92(0.59)$ & $0.53(0.19)$ & $0.77(0.05)$ & $1.19(0.12)$ \\
\hline Fourth & $1.38(0.20)$ & $0.66(0.11)$ & $0.74(0.17)$ & $0.61(0.45)$ & $1.08(0.66)$ & $1.04(0.84)$ \\
\hline Fifth and above & $1.17(0.56)$ & $1.35(0.23)$ & $1.57(0.03)$ & $0.89(0.86)$ & $1.01(0.98)$ & $1.64(0.03)$ \\
\hline \multicolumn{7}{|l|}{ Anaemia } \\
\hline Anaemic mothers & Ref. & Ref. & Ref. & Ref. & Ref. & Ref. \\
\hline Mother not anaemic & $0.83(0.07)$ & $0.86(0.18)$ & $0.85(0.07)$ & $0.82(0.47)$ & $1.02(0.78)$ & $1.06(0.44)$ \\
\hline \multicolumn{7}{|l|}{ Body mass index } \\
\hline Normal & Ref. & Ref. & Ref. & Ref. & Ref. & Ref. \\
\hline Underweight & $1.25(0.07)$ & $1.09(0.49)$ & $1.34(0.0)$ & $1.85(0.03)$ & $1.14(0.12)$ & $1.15(0.07)$ \\
\hline Overweight & $0.98(0.89)$ & $0.62(0.02)$ & $0.78(0.19)$ & $1.16(0.774)$ & $1(0.98)$ & $0.99(0.92)$ \\
\hline \multicolumn{7}{|l|}{ Working status } \\
\hline Non-working & Ref. & Ref. & Ref. & Ref. & Ref. & Ref. \\
\hline Working & $0.93(0.60)$ & $0.81(0.14)$ & $0.91(0.41)$ & $1.22(0.52)$ & $0.84(0.05)$ & $1.01(0.92)$ \\
\hline
\end{tabular}

in institutions were $46 \%$ more likely to give birth to LBW infants than those who delivered at home. Respondents in their fifth birth and above were $57 \%$ more likely to deliver LBW infants than those in their firth birth. Underweight mothers were $34 \%$ more likely to deliver LBW infants than mothers of normal weight. In the northeastern region, underweight mothers were $85 \%$ more likely to deliver LBW infants than mothers of normal weight.

In the western region, respondents with secondary education and above were $35 \%$ less likely to deliver
LBW infants than those uneducated. Respondents in their second and third births (28\% and $23 \%$ respectively) were less likely to deliver LBW infants than those in their first birth.

In the southern region, rural respondents were $25 \%$ more likely to deliver LBW infants than their urban counterparts. Respondents educated up to secondary level and above were $41 \%$ less likely to deliver LBW infants than those uneducated. Respondents within the age group of $35-49$ years were $45 \%$ less likely to deliver LBW infants than those in the age group of 15-24 years. 
RESEARCH ARTICLES

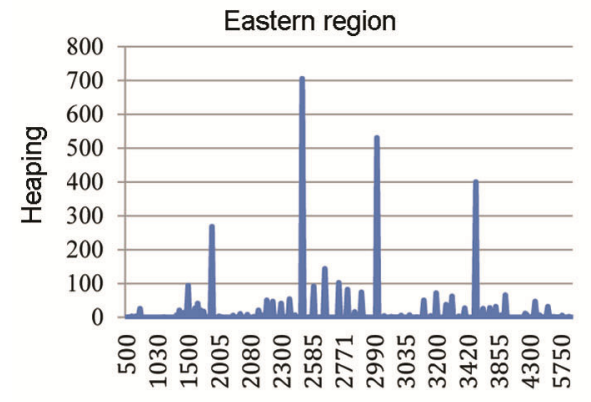

Birth weight

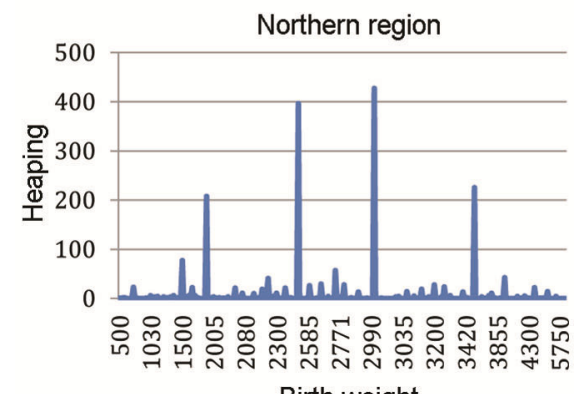

Birth weight

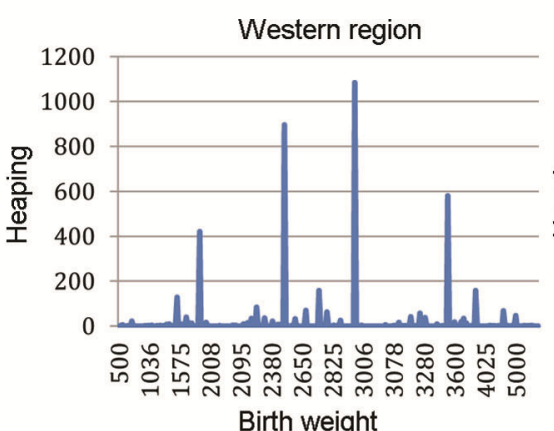

Birth weight

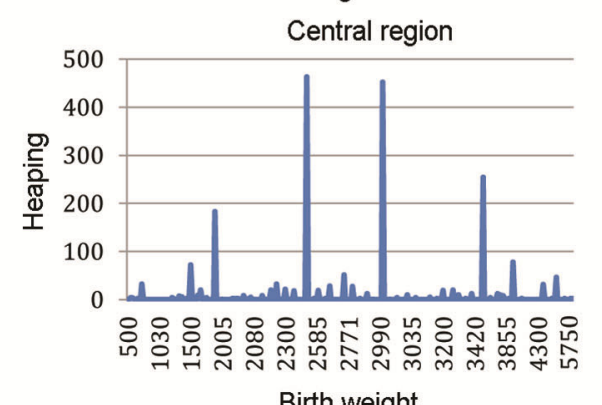

Birth weight
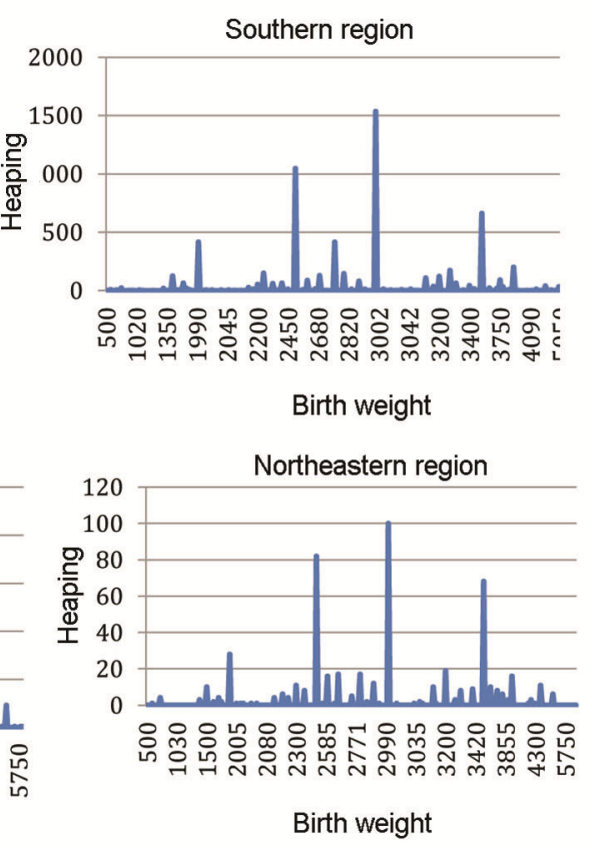

Figure 1. Heaping patterns of reported birth weights as multiples of $500 \mathrm{~g}$ for specific regions of India on the basis of NFHS: 2005-06 data.
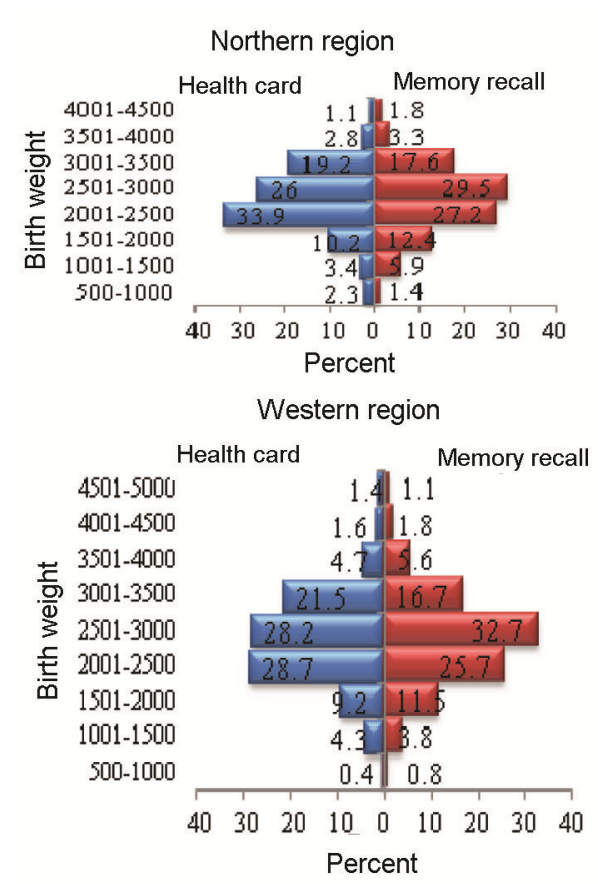

Southern region

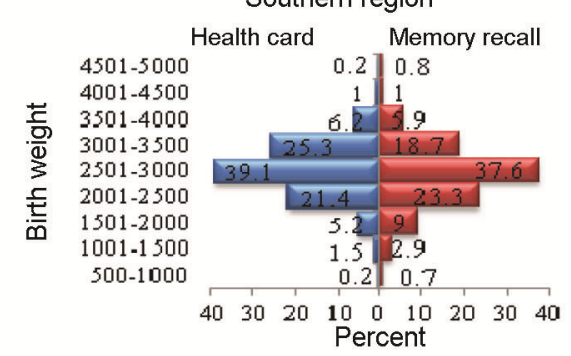

Central region

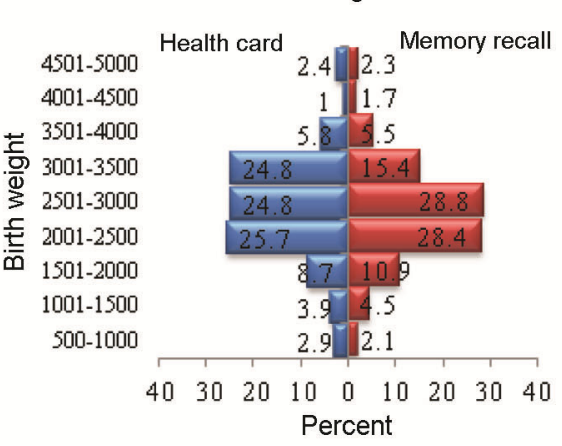

Eastern region

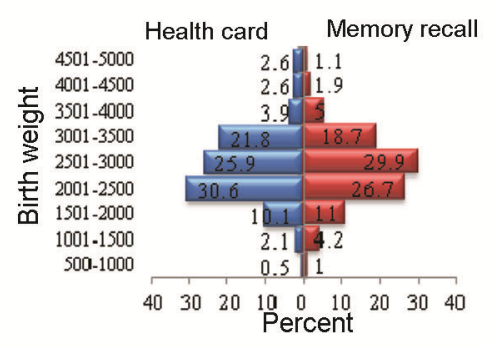

Northeastern region

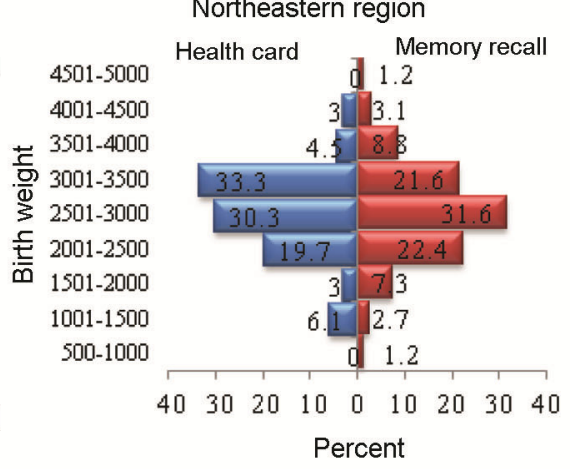

Figure 2. Pyramids of reported birth weights through mother memory recall and health card for specific regions of India on the basis of NFHS: 2005-06 data.

\section{Region-wise birth weight heaping}

In NFHS-3, data on numerical birth weight displayed considerable heaping on digits, which were multiples of $500 \mathrm{~g}$ in every region of India. Heaping refers to a pattern of misreporting in which the distribution of a number reported by respondents, such as age or birth weight, shows implausibly large frequencies of particular values, usually ending in 0 or 5 . Figure 1 shows the frequency distribution of birth weight for every region in India, in which heaping can be clearly observed. The heaping indicates that birth weights are often rounded-off to the nearest whole number, either by the medical personnel who weigh the infants and report the weight to the mother, or by mothers themselves when recalling the figures. This pattern suggests that there is some level of 


\section{RESEARCH ARTICLES}

incapacity in the mothers' recall of the exact birth weight with time and increase in the weight of the infant. In addition, heaping is substantially worse (i.e. there are more birth weights that are multiples of $500 \mathrm{~g}$ ) for infants whose weights have been reported by their mothers by the recall method, than when birth weights were recorded on a health card ${ }^{2}$.

\section{Region-wise birth-weight pyramids}

Figure 2 shows heaping patterns graphically demonstrated in the form of birth-weight pyramids for every region of India, which are similar to the population pyramids. These pyramids clearly prove that regional heaping is demonstrated through memory recall and documentation in health cards. Although the distribution of reported birth weights by health cards in all regions is not perfectly normal, it is approximately close to the unimodal distribution of weights. Report of birth weight through health cards in the northern, southern, eastern, western, central and northeastern regions is different, although there is evidence of heaping on certain weights. However, the weights recorded from health cards in all regions are highly heaped, and significantly different from those obtained through memory recall. The pyramids also specify that larger proportion of infants are within the intense distribution of birth weights (below and exactly $2500 \mathrm{~g}$ ), recalled from memory and the health card. This is conspicuous for all regions.

\section{Conclusion}

The profiling in this study suggests that across all the regions, the mothers were predominantly from rural areas, belonged to the middle age group, practised Hinduism, had their first births, were anaemic but with normal body mass index and were housewives. The prevalence of the region-specific distribution reporting of birth weight revealed that across all the regions, more mothers were from the urban areas, were educated up to secondary level and above, were in the middle age group (except in the eastern region), were more economically stable, belonged to the general caste category, delivered in institutional settings, had fewer births, were non-anaemic but unemployed and overweight.

Moreover, the region-specific distribution of prevalence of LBW revealed that across all the regions, proportionately higher respondents were from rural areas, with low level of education in the younger age group, were economically deprived, delivered at home, had experienced more births (except in the central and northeastern region), were anaemic and underweight.

The multivariable logistic regression model demonstrates a significant association between the reporting of birth weight and background characteristics, with education (secondary and above) and high economic stability (richer). Furthermore, higher the parity, lower is the odds for reporting of birth weight in the northern, central and eastern regions. Contrary to the above findings, majority of the background characteristics did not predict significant odds for low birth weight. This study can be explored further using the latest dataset.

Ethical approval: There is no ethical issue involved in this study as data are available in public domain for the purpose of research. Informed consent was obtained from all the respondents before conducting the interviews.

Competing interests: The authors have no competing interests.

1. Channon, A. A., Padmadas, S. S. and McDonald, J. W., Measuring birth weight in developing countries: does the method of reporting in retrospective surveys matter? Matern. Child Health J., 2011, 15(1), 12-8.

2. Blanc, A. K. et al., Monitoring low birth weight: an evaluation of international estimates and an updated estimation procedure. Bull. WHO, 2005, 83, 178-185.

3. Gagan, A. et al., Maternal risk factors associated with low birth weight neonates in a tertiary care hospital, Northern India. $J$. Community Med. Health Educ., 2012, 2, 177.

4. Kaushal, S. K. et al., A study of maternal factor and birth weight in a border district of Uttar Pradesh: a hospital based study. Indian J. Community Health, 2012, 24(2).

5. Shahnawaz et al., Association between maternal sociodemographic factors and low birth weight new born in a rural area of Bihar, India. South East Asia J. Public Health, 2014, 4(1), 3034.

6. Borah, M. and Baruah, R., Physical growth of low birth weight babies in first six month of life: a longitudinal study in a rural block of Assam. Natl. J. Commun. Med., 2014, 5(4), 397-400.

7. Dalal, A. et al., Epidemiological determinants of low birth weight in Ahmadabad city: a facility based case control study. Int. J. Med. Public Health, 2014, 3(4).

8. Sahu, K. K. et al., Incidence of low birth weight and effect of maternal factors on birth weight of neonates in rural areas of Uttar Pradesh. Int. J. Multidiscip. Res. Develop., 2015, 2(3), 707-715.

9. Noor, N., Kural, M., Joshi, T., Pandit, D. and Patil, A., Study of maternal determinants influencing birth weight of new born. Arch. Med. Health Sci., 2015, 3, 239-243.

10. Patel et al., Factors associated with low birth weight: community based retrospective study in an urban slum of Western India, 2015, vol. 1, issue 3; doi:10.5455/amr.20150504014512.

11. Kandhasamy and Singh, Determinants of low birth weight in a rural area of Tamil Nadu, India: a case-control study. Int. J. Med. Sci. Public Health, 2015, 4(3).

12. DHS, Demographic and Health Surveys, Calverton, MD, 2009.

13. IIPS, National Family Health Survey, 2005-2006: India. International Institute for Population Sciences, Mumbai, 2007.

ACKNOWLEDGEMENTS. The work described in this paper was extracted from Ph D thesis from Department of Statistics, Gauhati University, Assam, India.

Received 20 July 2018; revised accepted 21 February 2019

doi: $10.18520 / \mathrm{cs} / \mathrm{v} 116 / \mathrm{i} 10 / 1674-1682$ 